Original

\title{
Alternativa terapéutica para el dolor pélvico crónico de origen vesical
}

Pedro A. López González, Jesús I. Tornero Ruiz, Pedro López Cubillana, Gloria Doñate Iñiguez, Jose C. Ruíz Morcillo, Mariano Pérez Albacete

Servicio de Urología. Hospital Universitario Virgen de la Arrixaca. El Palmar. Murcia. España

\begin{abstract}
Resumen
Introducción: $\mathrm{El}$ dolor pélvico crónico (DPC) es un problema complejo e importante tanto por su frecuencia como por su morbilidad. Cuando se presenta asociado con síntomas miccionales deberemos pensar en un origen vesical.

Objetivo: Evaluar la respuesta a un tratamiento intravesical, al que denominamos Formula Sedante, en pacientes con dolor pélvico crónico de origen vesical, refractarios a otros tratamientos.

Material y métodos: Entre junio 2005 y septiembre de 2008, 14 pacientes (edad media de 59 años), 12 mujeres y 2 hombres, con DPC de origen vesical siguieron un tratamiento intravesical con "Formula Sedante", como terapia de segunda línea. Su composición es Dexametasona, Nitrofurantoína, Lidocaína y Suero salino. Evaluamos el dolor (escala 0-10), la frecuencia miccional y la mejoría subjetiva del paciente.

Resultados: El dolor global medio fue de 6’4 de base, al mes y a los 6 meses fue de 4’7 y 3’5 respectivamente. El 75\% de los pacientes mostró una disminución de los valores en la escala de dolor. La frecuencia miccional diurna mejoró un $28 \%$ al mes del tratamiento y un 40\% a los 6 meses. Un $70 \%$ de los pacientes refirió sentir mejoría tras la pauta de instilaciones, de los cuales en torno a un $80 \%$ la evaluó como "moderadamente mejor" o "mucho mejor", tanto al mes como a los 6 meses del tratamiento. Ninguno de los pacientes presento efectos secundarios reseñables.

Conclusiones: Consideramos el tratamiento de instilación intravesical con "Formula Sedante" una opción útil y segura, como segunda línea de tratamiento, para el dolor pélvico crónico de origen vesical.
\end{abstract}

Palabras clave: Dolor pélvico crónico. Cistitis intersticial. Tratamiento médico.

\section{New approach in treatment of pelvic chronic pain syndrome (CPPS)}

\section{Abstract}

Introduction: Chronic pelvic pain syndrome (CPPS) is a complex problem that is a major cause of morbidity and disability.

Objetive: To evaluate the feasibility, safety and efficacy of intravesical instillation of a new therapeutic tool in patients suffering from CPPS.

Methods: Between june 2005 and september 2008 we recruited 14 patients (mean age 59) who had been diagnosed with CPPS, and refractory to previous treatments. The intravesical solution consisted of steroids, local anaesthetic, antibiotic and saline solution. Was evaluated the intensity of pain; voiding frequency and subjective improvement (with patient global improvement impression PGI-I).

Results: Global pain at baseline was 6,4, at 1 month and 6 months 4,7 and 3,5. 75\% of the patients showed improvement in the reduction of pain. Voiding frequency at baseline was 22 '5 daily. At one and six months, voiding frequency was 16,2 and 13,5 respectively. According to the PGI-I test 30\% of patients were much better, 30\% moderate improvement, $10 \%$ slight improvement and 30\% with no changes at 1 month. At 6 months, $15 \%$ of patients were much better, $40 \%$ moderated improvement, $15 \%$ slight improvement and 30\% with no changes. None of the patients felt worse than before treatment. Conclusions: Therapeutic results of our intravesical solution traduces in an improvement of pain and voiding frequency reduction with no side-effects. Patients benefit of this improvement in the PGI-I test that reflects an improvement in quality of life. We consider this treatment a second-line approach for CPPS in which gold standard management is still a challenge.

Keywords: Pelvic pain. Interstitial cystitis.Treatment outcome.

$\mathrm{E}$ dolor pélvico crónico (DPC) afecta fundamentalmente a mujeres y se define por una duración superior a 6 meses, en ausencia de patología infecciosa y/o tumoral. Es un problema importante tanto por su frecuencia como por su morbilidad, de forma que actualmente se piensa que el
DPC puede afectar a más del 15\% de las mujeres, y que al menos 1 de cada 3 experimentará este cuadro alguna vez en su vida. La incidencia de depresión en estas pacientes es del 30-54 \% comparada con las tasas normales del 5-17\% en la población general. 
A menudo se asume que el DPC es de origen ginecológico, pero es esencial pensar más allá de los órganos reproductivos y considerar que cada estructura del abdomen y/o de la pelvis puede tener un papel en la etiología del DPC, y entre estas la vejiga. Además no debemos olvidar que este cuadro también puede afectar a varones ${ }^{1,2}$.

Las causas del dolor pélvico crónico son muy diversas, destacando según su origen ${ }^{3}$ :

a) Ginecológicas: endometriosis, síndrome de congestión pélvica,...

b) Urológicas: cistitis intersticial, prostatitis y epididimitis crónica, patología del suelo pélvico, disinergia esfinteriana,...

c) Gastrointestinales: Síndrome de intestino irritable, enfermedad inflamatoria intestinal, estreñimiento crónico,...

d) Musculoesqueléticas

e) Neurológicas

f) Psiquiátricas.

Para el esclarecimiento del origen del DPC las herramientas fundamentales son una historia clinica detallada, haciendo énfasis en las características del dolor y los síntomas acompañantes, y un completo examen físico ${ }^{4,5}$.

Como hemos subrayado el dolor pélvico crónico puede tener su origen en la vejiga, acompañándose de otros síntomas como frecuencia urinaria y urgencia miccional.

Dentro de este grupo destaca, sin lugar a dudas, tanto por su complejidad como por su frecuencia, la Cistitis Intersticial.

El tratamiento de esta patología es normalmente inespecífico, empírico, no curativo, y cuya meta es el alivio de los sintomas. La existencia de una gran variedad de modalidades terapéuticas es reflejo de la pobre eficacia de éstas, siendo a menudo necesario la combinación o el uso alternativo de varias de ellas. Algunos de los tratamientos utilizados son:

Medidas generales: Supresión de comidas que pueden incrementar los sintomas como la cafeína, alcohol y las comidas ácidas ${ }^{6}$, fisioterapia ${ }^{7}$.

Terapia oral: Antidepresivos tricíclicos (Amitriptilina, Doxepina, Imipramina): Son fármacos eficaces en bloquear el inicio del dolor ${ }^{8,9}$; Antihistamínicos (Hidroxicina y Cimetidina) ${ }^{10}$; Pentosan-polisulfato de sodio (Elmiron $\left.{ }^{\circledR}\right)^{11,12}$; Prednisona, ha demostrado su utilidad pero con presencia de efectos secunda- rios importantes ${ }^{13}$. Otros tratamientos orales usados son la Gabapentina, AINEs, antagonistas del calcio (Nifedipino), inmunosupresores (Azatriopina), anticolinérgicos. A veces se combinan dos o más terapias orales para mejorar la respuesta, como el caso de la Amitriptilina con Pentosan Polisulfato de sodio e Hidroxicina.

Terapia intravesical: Dimetil sulfóxido (DMSO) ${ }^{14} \mathrm{y}$ ácido hialurónico, ambos tratamientos como "reponedores" de glicosaminoglicanos ${ }^{15}$. Otros tratamientos menos utilizados incluyen Heparina, Oxicloroseno, Capsaicina, Resiniferatoxina, Lidocaína, BCG (Bacillus Calmette Guerin) ${ }^{16}$, Cromoglicato Sódico y Orgoteína.

Otras modalidades de tratamiento: Distensión vesical, con balón o hidráulica; neuromodulación ${ }^{17}$; y el tratamiento quirúrgico, como la cistectomía parcial o radical, reservado para casos graves refractarios a todas la modalidades de tratamiento expuestas anteriormente ${ }^{18}$.

\section{OBJETIVO}

Evaluar la respuesta a un tratamiento intravesical al que denominamos Formula Sedante en pacientes con dolor pélvico crónico de origen vesical, refractarios a otros tratamientos.

\section{MATERIAL Y MÉTODOS}

Entre junio 2005 y septiembre de 2008, 14 pacientes (12 mujeres y 2 hombres), con edad media de 59,6 años (27-78 años), y con presencia de dolor pélvico crónico de origen vesical, fueron sometidos a un protocolo de instilaciones intravesicales con una solución a la que denominamos Formula Sedante.

Entre sus antecedentes personales destacan por su frecuencia la existencia de alergia (21\%), tratamiento crónico con ansiolíticos y/o antidepresivos (57\%), migraña (28\%) y patología articular (43\%). Dos de las pacientes habían sido sometidas a cirugía vesical, una por reflujo vesico-ureteral en la infancia y otra a ileocistoplastia de ampliación, cinco años antes del tratamiento endovesical. Todos los pacientes referían dolor pélvico localizado en región suprapúbica, excepto un caso que se localizaba en zona perineal, con una evolución media de 8,3 años. Los síntomas acompañantes incluían urgencia miccional (73\%) e incontinencia urinaria (35\%) 
A todos los pacientes se les realizó un urocultivo, citología y cistoscopia con resultado negativo, descartando la existencia de infección del tracto urinario y de neoplasia vesical. Otras exploraciones complementarias, como estudio urodinámico, ecografía, radiología simple y $\mathrm{TAC}$, se realizaron en casos seleccionados según la clínica del paciente. Además se realizó una biopsia vesical en 4 ocasiones por sospecha de carcinoma in situ, siendo negativa en todos los casos.

El 72\% de ellos fue tratado con fármacos anticolinérgicos, el 20\% recibió alfa-bloqueantes, el 36\% tratamiento antibiótico empírico, 15\% recibieron gabapentina y 10\% hidroxicina. En algunos casos de forma combinada, sin conseguir en ninguno de los casos una respuesta satisfactoria.

Para evaluar la eficacia del tratamiento intravesical valoramos:

\section{Dolor}

Lo evaluamos según la escala numérica de Downie, siendo el 0 la ausencia de dolor, y el 10 el peor dolor imaginable. En algunos casos seleccionados utilizamos una escala visual analógica. Diferenciamos el dolor según tenga lugar durante la fase de llenado vesical o la de vaciado. Se evaluó el nivel de dolor del paciente antes del tratamiento, al mes y a los seis meses de su finalización.

\section{Frecuencia miccional}

Lo evaluamos a través de un diario miccional. Diferenciamos frecuencia miccional diurna y nocturna. Se evaluó la frecuencia miccional antes del tratamiento, al mes y a los seis meses de su finalización.

\section{Mejoria subjetiva}

Lo evaluamos según la siguiente escala de Impresión de Mejoría Global del Paciente (PGI-I):

0. No evaluado

1. Mucho mejor

2. Moderadamente mejor

3. Levemente mejor

4. Sin cambios

5. Levemente peor

6. Moderadamente peor

7. Mucho peor

El tratamiento endovesical utilizado, "Formula Sedante", tiene la siguiente composición: Dexametasona $40 \mathrm{mg} / 5 \mathrm{ml}$, Nitrofurantoína susp. $50 \mathrm{mg} / 5 \mathrm{ml}$,
Lidocaína $100 \mathrm{mg} / 5 \mathrm{ml}$, Suero salino 0,9\%/35 ml. Se realiza sondaje vesical $y$, tras vaciar vejiga, se procede a la administración de los $50 \mathrm{ml}$ de la solución. El paciente debe de mantener la solución en la vejiga durante 1 hora.

El protocolo de tratamiento, aunque puede ser variable, es el siguiente:

- 3 veces/semana durante 2 semanas.

- 2 veces/semana durante las 4 semanas siguientes.

\section{RESULTADOS}

Hubo un abandono tras la primera instilación debido a que la paciente no pudo retener la solución intravesicalmente más de 5 minutos.

En ninguno de los casos se presentaron efectos secundarios significativos, solamente leves molestias asociadas a la instilación.

\section{Dolor}

Antes del tratamiento los pacientes refirieron, como media, un nivel de dolor al llenado vesical y durante la micción de 6,5 y 6,2 respectivamente (dolor global medio: 6,4). Al mes y a los 6 meses se encontraron los siguientes datos: Dolor medio al llenado vesical de 4,5 al mes y 4,1 a los seis meses; dolor medio durante la micción de 4,8 y 3,0 al mes $\mathrm{y}$ a los seis meses respectivamente (dolor global medio de 4,7 al mes y 3,5 a los seis meses). El 75\% de los pacientes mostraron una reducción en sus niveles de dolor con una mejora media del 43\% al mes y del 34\% a los 6 meses (Tabla 1).

Tabla 1. Intensidad del dolor

\begin{tabular}{lccc}
\hline & $\begin{array}{c}\text { Dolor al } \\
\text { llenado }\end{array}$ & $\begin{array}{c}\text { Dolor al } \\
\text { vaciado }\end{array}$ & $\begin{array}{c}\text { Dolor } \\
\text { global }\end{array}$ \\
\hline Basal & 6,5 & 6,2 & 6,4 \\
Al mes & 4,5 & 4,8 & 4,7 \\
A los 6 meses & 4,1 & 3 & 3,5 \\
\hline
\end{tabular}

\section{Frecuencia miccional}

Antes del tratamiento los pacientes presentaban una frecuencia miccional diurna media de 22,5 veces y una frecuencia miccional nocturna media de 5 veces. Tras el tratamiento refirieron una frecuencia miccional diurna media de 16,2 y 13,5 veces al mes y a los 6 meses respectivamente; y una frecuencia miccional nocturna media de 4,1 al mes y de 5 veces a los 6 meses. Esto supone una mejora 
del $28 \%$ al mes y del $40 \%$ a los 6 meses, en la frecuencia miccional diurna. En cuanto a la frecuencia miccional nocturna, apenas se apreciaron cambios (Tabla 2).

Tabla 2. Frecuencia miccional

\begin{tabular}{lcc}
\hline & $\begin{array}{c}\text { Fr Miccional } \\
\text { diurna }\end{array}$ & $\begin{array}{c}\text { Fr Miccional } \\
\text { nocturna }\end{array}$ \\
\hline Basal & 22,5 & 5 \\
Al mes & 16,2 & 4,1 \\
A los 6 meses & 13,5 & 5 \\
\hline
\end{tabular}

\section{Mejoria subjetiva}

Según la escala utilizada, para medir la impresión de mejoría del paciente, los resultados al mes de finalizar el tratamiento fueron: $30 \%$ mucho mejor; 30\% moderadamente mejor, 10\% levemente mejor y 30\% sin cambios. A los 6 meses los datos fueron los siguientes: $15 \%$ mucho mejor, $40 \%$ moderadamente mejor, 15\% levemente mejor, 30\% sin cambios. En ningún caso los pacientes refirieron encontrarse peor que al inicio del tratamiento (Tabla 3).

\section{DISCUSIÓN}

Decidimos rescatar un tratamiento endovesical, al que denominamos "Formula Sedante", para el tratamiento del dolor pélvico crónico de origen vesical, como terapia de segunda línea. La farmacoterapia intravesical proporciona unas altas concentraciones locales de fármaco en la vejiga, evitando los efectos secundarios sistémicos y eliminando el problema de los bajos niveles de excreción urinaria con los agentes administrados oralmente. Esto era especialmente importante con la utilización de corticoides, ya que aunque habían demostrado ser eficaces por via oral en casos refractarios, sus efectos adversos los hacían poco útiles ${ }^{13}$. Con respecto a los demás componentes, la lidocaína mejora la tolerabilidad de la solución y la nitrofurantoína proporciona una profilaxis infecciosa ante la manipulación en la vía urinaria.
Los resultados que hemos detallado confirman una eficacia significativa, tanto en la reducción del dolor como en la mejora de la frecuencia miccional diurna. Además es reseñable que respondieron un $75 \%$ de los pacientes. Pero quizás lo más significativo son los resultados de mejoría subjetiva del paciente. En éstos se pone de relieve que la mayoría de los pacientes (70\%) presentaron una mejora significativa en su calidad de vida, siendo éste, sin duda, el objetivo último de cualquier tratamiento utilizado para el dolor pélvico crónico.

Otros aspectos que quedan por definir son: el régimen de tratamiento óptimo; si existe una correlación entre la dosis de tratamiento y la respuesta de los pacientes; y si es pertinente un tratamiento de mantenimiento, en pacientes con buenos resultados. Para responder a estas cuestiones se necesita una muestra más amplia, no siendo el objetivo de este trabajo.

Destacamos la comorbilidad de los pacientes en estudio, sobre todo la elevada frecuencia de alergia, tratamiento crónico con ansiolíticos y/o antidepresivos, migraña y patología articular. Estos datos coinciden con la patología asociada a cistitis intersticial, según la bibliografía consultada.

\section{CONCLUSIONES}

Dados los resultados obtenidos, y la ausencia de efectos secundarios significativos, podemos concluir que el tratamiento endovesical con "Formula Sedante" es eficaz y seguro, como terapia de $2^{\text {a }}$ línea, para el dolor pélvico crónico de origen vesical.

\section{REFERENCIAS}

1. Burkman RT. Chronic pelvic pain of bladder origin: epidemiology, pathogenesis and quality of life. J Reprod Med. 2004;49(3 Suppl):225-229.

2. Gunter J. Chronic pelvic pain: an integrated approach to diagnosis and treatment. Obstet Gynecol Surv. 2003;58(9):615-623.

3. Hakenberg OW, Wirth MP. Chronic pelvic pain in men. Urol Int. 2002;68(3): 138-143.

4. Martin D, Ling F. Endometriosis and pain. Clin Obstet Gynecol. 1999;42(3):664-692.

5. Barlow DH, Glynn CJ. Endometriosis and pelvic pain. Ballieres Clin Obstet Gynecol. 1993;7(4):775-789.

6. Whitmore KE. Self-care regimens for patients with interstitial cystitis. Urol Clin North Am. 1994;21(1):121-130.

Tabla 3. Mejoría subjetiva. Impresión de Mejoría Global del Paciente (PGI-I)

\begin{tabular}{lcccc}
\hline PGI-I & Mucho mejor & Moderadamente mejor & Levemente mejor & Igual \\
\hline Al mes & $30 \%$ & $30 \%$ & $10 \%$ & $30 \%$ \\
A los 6 meses & $15 \%$ & $40 \%$ & $15 \%$ & $30 \%$ \\
\hline
\end{tabular}


7. Weiss J. Pelvic floor myofascial trigger points: Manual therapy for interstitial cystitis and the urgency-frequency syndrome. J Urol. 2001;166(6):2226-2231.

8. Pranikoff $\mathrm{K}$, Costantino G. The use of amitriptyline in patients with urgency, frequency and pain. Urology. 1998;51(suppl 5): 179-181.

9. Hanno PM. Amitriptyline in the treatment of interstitial cystitis. Urol Clin North Am.1994;21(1):89-91.

10. Theoharides TC, Sant GR. Hydroxyzine therapy for interstitial cystitis. Urology.1997;49(suppl 5ª):108-110.

11. Hanno PM. Analysis of long-term Elmiron therapy for interstitial cystitis. Uology.1997;49(suppl 5ª):93-99.

12. Nickel JC, Barkin J, Forrest J, et al. Randomized, double-blinded, dose-ranging study of pentosan polysulfate sodium for interstitial cystitis. J Urol.2001;165(5 suppl):67.

13. Soucy F, Gregoire M. Efficacy of prednisone for severe refractory ulcerative interstitial cystitis. J Urol. 2005;173(3):841-843.

14. Parkin J, Shea C, Sant GR. Intravesical dimethyl sulfoxido (DMSO) for interstitial cystitis-A practical approach. Urology.1997;49(Suppl 5A): 105-107.

15. Morales A, Emerson L, Nickel JC, Lundie M. Intravesical hyaluronic acid in the treatment of refractory interstitial cystitis. J Urol. 1996;156(1):45-48.
16. Peters K, Diokno A, Steinert B, Yuhico M, Mitchell B, Krohta S, et al. The efficacy of intravesical Tice srain bacillus CalmetteGuerin in the treatment of interstitial cystitis:a double blind, prospective, placebo controlled trial. J Urol. 1997;157(6):20902094.

17. Siegel S, Paszkiewicz E, Kirkpatrick C, Hinkel B, Oleson K. Sacral nerve stimulation in patients with chronic intractable pelvic pain. J Urol. 2001;165(5):1742-1745.

18. Cózar Olmo JM, Martínez Morcillo A, Vicente Prados J, Tallada Buñuel M, Espejo Maldonado E. Cistitis intersticial: actualización de su patogenia, diagnóstico y tratamiento. Arch Esp Urol. 2002;55(9):1153-1164.

Correspondencia autor: Pedro A. López González Servicio de Urología

Hospital Universitario Virgen de la Arrixaca

Ctra. Madrid-Cartagena, s/n - 30120 El Palmar. Murcia Tel.: 968369500

E-mail autor: peterangel@ono.com

Información artículo: Original - Dolor pélvico

Trabajo recibido: enero 2009

Trabajo aceptado: febrero 2009 